\title{
Partial cross sections for dissociative electron attachment to tetrahydrofuran reveal a dynamics-driven rich fragmentation pattern
}

\author{
R. Janečkováa ${ }^{a}$ O. May ${ }^{a}$, A.R. Milosavljević ${ }^{b}$, J. Fedor $^{\mathrm{a}, *}$ \\ a Department of Chemistry, University of Fribourg. Chemin du Musée 9, CH-1700 Fribourg, Switzerland \\ ${ }^{\mathrm{b}}$ Institute of Physics Belgrade, University of Belgrade, Pregrevica 118, 11080 Zemun, Belgrade, Serbia
}

\section{Introduction}

Electron collisions with tetrahydrofuran (THF) attracted a surprising amount of attention during the last 15 years. This interest which may seem unusual for a small organic molecule - has been part of the line of research in electron collisions with molecules of biological relevance. This line of research aims at elucidating the role of electron-triggered processes in radiation damage. In this respect THF, since it is the simplest saturated furanose ring, can be considered as a prototype for the DNA sugar backbone. Additionally, in contrary to sugars, THF is a liquid at standard laboratory conditions which greatly facilitates the experiments.

The above-mentioned line of research has been triggered by a ground breaking discovery of Sanche and co-workers [1] that lowenergy electrons cause single and double strand DNA breaks even at sub-ionization energy. Inspired by this discovery, various research groups started to look at the electron-induced reactions in molecular constituents of DNA. One of the first groups that adopted this direction - and quickly became a beacon in the field - was the group of T.D. Märk in Innsbruck. Märk recognized the high scientific potential in this field and the experiments in his group have greatly contributed to a renewal of the interest in electron-triggered

\footnotetext{
* Corresponding author. Tel.: +41 300268697

E-mail address: juraj.fedor@unifr.ch (J. Fedor).
}

physics and chemistry, especially the dissociative electron attachment (DEA). This process, which proceeds via formation of a transient resonant state is an effective bond-breaking reaction and the experiments in Innsbruck have elucidated its role in a whole range of molecules with biological relevance: DNA bases [2,3], sugars [4], or amino acids [5]. The experiments have revealed a plethora of phenomena occurring in dissociative electron attachment: siteand bond-selectivity [6] or DEA footprints of vibrational Feshbach resonances in biomolecules [7]. The group of T.D. Märk has been also the first one that looked into DEA process in the molecule of present interest: tetrahydrofuran [8].

Basically all experimentally accessible electron-triggered processes in THF have been characterized in the recent years. The total electron scattering cross sections were reported by Zecca et al. [9], Mozejko et al. [10] and Fuss et al. [11]. The elastic scattering in various energy ranges was probed by Milosavljević et al. [12], Dampc et al. [13], Allan [14], Colyer et al. [15], Baek et al. [16], Chiari et al. [17] and Homem et al. [18]. The vibrational excitation cross sections were reported by Dampc et al. [19] and Allan [14] and electronic excitation was probed by Do et al. [20] and Giuliani et al. [21]. The electron collisions with THF attracted also considerable amount of attention from a theoretical point of view. The scattering processes were characterized by a Schwinger multichannel method [22], complex Kohn variational method [23], or R-matrix method with Born correction [24]. The last study is of special interest here since it identified a number of core-excited resonances. Complementary to the gas phase, series of experiments with THF deposited 
on a surface was performed by Lepage et al. [25], Antic et al. [26,27], Breton et al. [28], Jäggle et al. [29], and Park et al. [30].

The dissociative electron attachment channels in THF have also been the subject of several studies, Sulzer et al. [8] and Ibanescu et al. [31] reported yields of individual DEA fragments as a function of the electron energy and Aflattoni et al. [32] measured total DEA cross section using a total ion collection instrument. Surprisingly, the first two studies only partially agree with each other: they agree on the occurrence of the (strongest) fragment with atomic mass 41 , which was assigned by both groups to be the $\mathrm{C}_{2} \mathrm{HO}^{-}$anion. However, Sulzer et al. also reported the occurrence of a parent anion $\mathrm{THF}^{-}$and a doubly dehydrogenated parent anion (THF-2H) ${ }^{-}$ around $2 \mathrm{eV}$, which were not detected by Ibanescu et al. who, on the other hand, reported hydride anion $\mathrm{H}^{-}$and anion with mass 43 $\left(\mathrm{C}_{2} \mathrm{H}_{3} \mathrm{O}^{-}\right)$. The occurrence of stable parent anions in DEA at energies considerably higher than $0 \mathrm{eV}$ is very rare, since the excess energy (electron affinity and incident electron energy) has to be stored within internal degrees of freedom. Such situation often leads to metastability of the parent anion in the microsecond time window [33,34]: the excitation energy randomizes over the internal degrees of freedom and the decay of the parent anion results from the concentration of sufficient energy in the relaxation channel.

Our primary motivation to study THF was the eventual occurrence of such metastability, which can be detected with a time-of-flight setup such as the one available in our laboratory. We report negative evidence for metastable processes in our time window, however, we have discovered several surprising aspects of the DEA to THF. The high sensitivity of our instrument enabled identification of a number of previously unreported anionic fragments, thus revealing much more complex fragmentation dynamics. We also provide new assignment of the strongest heavy anionic fragments with the mass 41 , which turned out to be $\mathrm{C}_{3} \mathrm{H}_{5}{ }^{-}$. We provide absolute partial DEA cross sections for all reported fragments and discuss the possible origins of resonant states leading to the reported dissociation pattern.

\section{Experiment}

The quantitative DEA spectrometer has been described in detail previously [35-37]. The electron beam is produced by a trochoidal electron monochromator, passes through a collision cell filled with the sample gas and is collected by a Faraday cup. Anions created by DEA are extracted through a narrow slit in the cell's wall to a time-of-flight mass (TOF) spectrometer. The experiment works in a pulsed mode. The electron beam passes through the collision cell for $200 \mathrm{~ns}$ while it is field-free. After another $200 \mathrm{~ns}$ (so that the electrons are allowed to leave the cell) a voltage pulse of $-300 \mathrm{~V}$ is applied across the cell to expel the anions. They are detected by a microchannel plate coupled with phosphorus screen and photomultiplier, counted and their arrival times are analyzed using a delayed coincidence scheme. The spectra are recorded as a twodimensional map of the ion signal as a function of electron energy and the flight time (which reflects the anion mass). The repetition rate is $50 \mathrm{kHz}$. The pressure in the collision cell is controlled by a needle valve and monitored by a capacitance manometer. It was kept in the range of $3 \times 10^{-4}$ to $6 \times 10^{-4}$ Torr. Since the previous studies did not reveal any fine structures in DEA ion yields, the electron monochromator was operated to yield high electron current (around $100 \mathrm{nA}$ in the $\mathrm{CW}$ mode) and thus a total high sensitivity. The electron energy resolution during the present measurements was $250 \mathrm{meV}$.

The TOF setup has two acceleration regions and a drift region. The first acceleration region is between the pulsed pusher and the wall of the collision chamber, the second acceleration region is between the wall of the chamber and the front plate of the time-of-flight tube. The TOF tube (drift region) is not entirely fieldfree, it is constructed as a focusing ion lens and the entire setup was designed to keep the extraction and detection efficiency independent of the mass and initial kinetic energy of the detected anions [37]. Additionally, its first part has a deflecting capacity in order to compensate for the effect of the magnetic field. It has been shown by simulations and extensive tests that only two types of voltage settings are necessary for the proper collection: 'light ion settings' for $\mathrm{H}^{-}$and $\mathrm{D}^{-}$ions and 'heavy ion settings' for ions with larger masses. The mass scale is calibrated with ions of known masses from other compounds $\left(\mathrm{O}^{-}\right.$from $\mathrm{O}_{2}, \mathrm{H}^{-}, \mathrm{C}_{2}{ }^{-}$and $\mathrm{C}_{2} \mathrm{H}^{-}$from acetylene) and the calibration is based on the fact that the time-of-flight is directly proportional to the square root of mass. The mass assignment is verified by the fact that all assigned masses fit perfectly on the calibration curve.

The electron energy scale was calibrated using the $4.4 \mathrm{eVO}^{-} / \mathrm{CO}_{2}$ peak. The shape of this band (with a threshold at $3.99 \mathrm{eV}$ ) has been measured with high electron-energy resolution [38]. We determine both the energy scale calibration and the electron energy resolution by fitting the convoluted high-resolution data to our measurement. Absolute magnitudes of the cross sections were calibrated on the same $\mathrm{O}^{-} / \mathrm{CO}_{2}$ band with a peak cross-section value of $14 \mathrm{pm}^{2}$ [35]. The error of the absolute measurements (two standard deviations) is taken to be $\pm 25 \%$. This is the combined error of $\pm 20 \%$ of the relative measurements with the $\pm 15 \%$ error of the $\mathrm{O}^{-} / \mathrm{CO}_{2}$ absolute cross section. We have shown recently [39] that this calibration approach is not appropriate if there are narrow bands in the DEA spectra due to effect of electron beam resolution on the peak width, however, it is perfectly suitable for the present molecule since only broad bands appear in the spectra (see below).

\section{Results and discussion}

\subsection{Anionic fragments and partial cross sections}

Fig. 1 (top) shows the negative ion mass spectrum of tetrahydrofuran originating from the impact of $8.5 \mathrm{eV}$ electrons. One can observe a rich fragmentation pattern with a number of previously unreported anionic fragments. The chemical composition of all

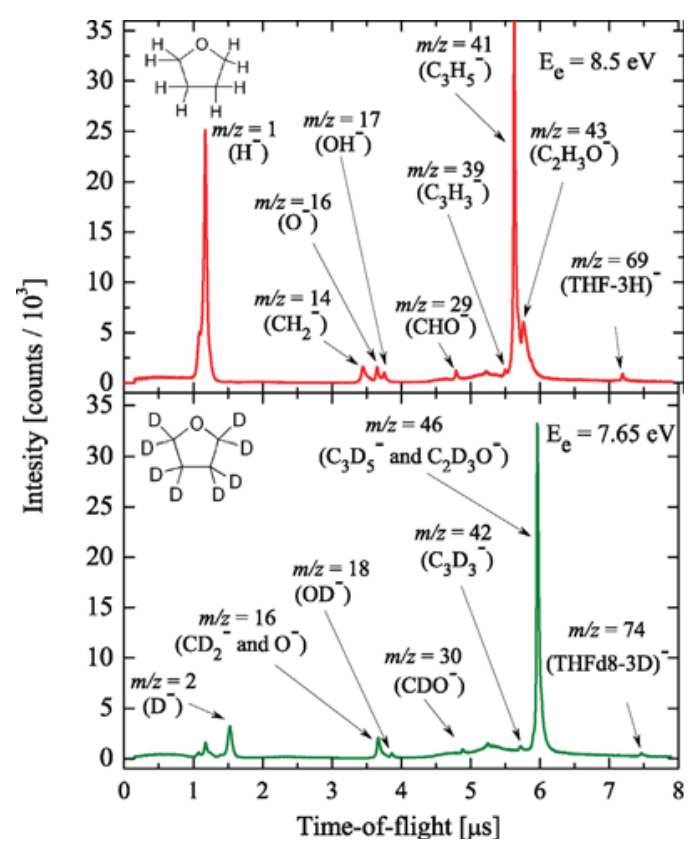

Fig. 1. Negative ion TOF mass spectrum of tetrahydrofuran (top) and fully deuterated tetrahydrofuran THF-d8 (bottom). 


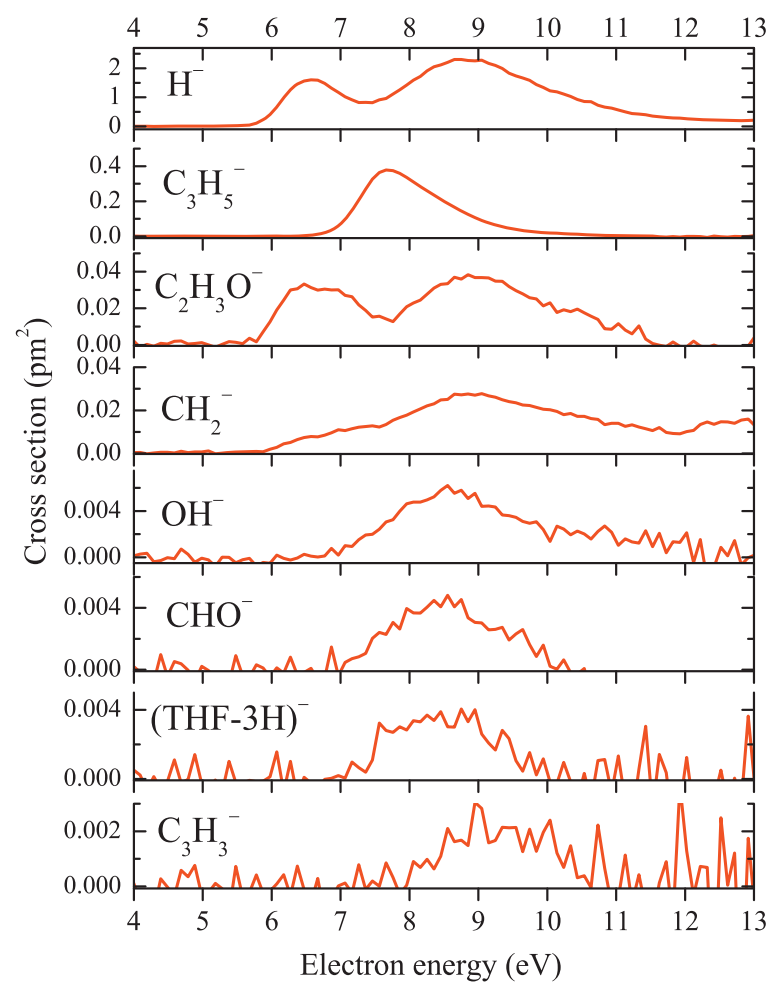

Fig. 2. Partial cross sections for production of anionic fragments in THF.

fragments was determined upon comparison with the mass spectrum of fully deuterated THF (Fig. 1 bottom). Fig. 2 shows the partial absolute cross sections for all fragments. In spite of a careful acquisition we did not detect any signal below $5 \mathrm{eV}$ electron energy. Note that the mass spectra in Fig. 1 were taken at heavy ion settings, thus the intensity of $\mathrm{H}^{-}$peak is not directly comparable with Fig. 2. This is also the reason for a shorter flight-time shoulder of the $\mathrm{H}^{-}$signal.

The previously unreported fragments are $\mathrm{CH}_{2}{ }^{-}, \mathrm{OH}^{-}, \mathrm{COH}^{-}$, $\mathrm{C}_{3} \mathrm{H}_{3}{ }^{-}$and $\mathrm{C}_{4} \mathrm{H}_{5} \mathrm{O}^{-}$[triply dehydrogenated tetrahydrofuran anion $(\mathrm{THF}-3 \mathrm{H})^{-}$]. The strongest heavy fragment with the mass 41 has been reported both by Sulzer et al. [8] and by Ibanescu et al. [31]. The shape and position of the band agrees very well with present results. Additionally, Ibanescu et al. reported $\mathrm{C}_{2} \mathrm{H}_{3} \mathrm{O}^{-}$and $\mathrm{H}^{-}$fragments. The position and shape of the bands again agree well with present results for $\mathrm{C}_{2} \mathrm{H}_{3} \mathrm{O}^{-}$and reasonably well for $\mathrm{H}^{-}$. In the latter case the relative intensities of the two bands show certain discrepancies. It should be noted that the two experiments are not directly comparable - Ibanescu et al. used a quadrupole mass filter where the transmission efficiency is known to depend strongly on the ion mass and on the initial kinetic energy. All the newly reported fragments except $\mathrm{CH}_{2}{ }^{-}$have very low cross sections below $0.01 \mathrm{pm}^{2}$ which is probably the reason for their absence in earlier studies. Comparison of the two mass spectra also shows that the peak with the mass 41 shifts to mass 46 upon deuteration of the precursor molecule. Thus its chemical composition is $\mathrm{C}_{3} \mathrm{H}_{5}{ }^{-}$, the allyl anion, instead of $\mathrm{C}_{2} \mathrm{OH}^{-}$, deprotonated ketene, as it was assigned in both previous mass-resolved studies [8,31]. The reason for their assignment was probably the fact that the formation of $\mathrm{C}_{2} \mathrm{OH}^{-}$is energetically more favorable.

Table 1 summarizes threshold energies for the reaction channels leading to production of anions with molecular mass $41 \mathrm{amu}$. The calculations were performed at the B3LYP/6-311++G(2df, 2pd) level of theory. This method has been shown to yield reliable asymptotic energies in a range of molecules [40,41]. It has been pointed out in number of theoretical investigations (see Ref. [21] for review), that neutral THF has several different conformations closely spaced
Table 1

DFT [B3LYP/6-311++G(2df, 2pd)] threshold energies $(\mathrm{eV})$ for various channels leading to production of anions with $m / z=41$.

\begin{tabular}{lll}
\hline Process & Products & $E_{\text {th }}(\mathrm{eV})$ \\
\hline $\mathrm{e}+\mathrm{THF} \rightarrow$ & $\mathrm{C}_{2} \mathrm{HO}^{-}+\mathrm{C}_{2} \mathrm{H}_{5}+\mathrm{H}_{2}$ & 1.84 \\
& $\mathrm{C}_{3} \mathrm{H}_{5}^{-}+\mathrm{CH}_{3} \mathrm{O}$ & 2.59 \\
& $\mathrm{C}_{3} \mathrm{H}_{5}^{-}+\mathrm{CH}_{2} \mathrm{O}+\mathrm{H}$ & 3.63 \\
\hline
\end{tabular}

in energy, all of them being connected via out-of-plane ring deformation (pseudorotation). The high-level ab-initio calculations [42] reveal that the two lowest conformers, with $C_{2}$ and $C_{s}$ symmetry, have only $6 \mathrm{meV}$ difference in energy, which results in almost equal Boltzmann population of these two structures in the gas phase. We have optimized the neutral THF within the $\mathrm{C}_{2}$ geometry. The calculated structure of $\mathrm{C}_{3} \mathrm{H}_{5}{ }^{-}$corresponds to allyl anion, the calculated structural formula of the $\mathrm{C}_{2} \mathrm{OH}^{-}$is $\mathrm{HCCO}^{-}$. As can be seen in Table 1 , the channel leading to $\mathrm{C}_{2} \mathrm{HO}^{-}+\mathrm{C}_{2} \mathrm{H}_{5}+\mathrm{H}_{2}$ is indeed the energetically most favorable. Both channels leading to production of $\mathrm{C}_{3} \mathrm{H}_{5}$ are higher in energy: the one leading to production of the methoxy radical $\mathrm{CH}_{3} \mathrm{O}$ by $0.75 \mathrm{eV}$, the one leading to production of formaldehyde and hydrogen atom by $1.79 \mathrm{eV}$. However, as one can see in Fig. 2, the $\mathrm{C}_{3} \mathrm{H}_{5}{ }^{-}$band starts at approximately $6.7 \mathrm{eV}$, with all channels easily accessible and it is not the asymptotic energetics but rather the dissociative direction of the involved resonant potential energy surface that determines the fragmentation pathway. This shows dominant role of the fast nuclear dynamics - the internal energy does not redistribute and seek the lowest energy pathway (as one could intuitively expect for such relatively large molecule) but rather follows the direct path on the potential energy surface.

The mass spectra in Fig. 1 also contain some spurious peaks. The small peak with mass 35 that can be discernible in both spectra is atomic chlorine anion $\mathrm{Cl}^{-}$which comes from interaction of electrons with trace amounts of chlorinated species that are present in the experimental setup from previous experiments. We also believe that a large part of the $\mathrm{O}^{-}$signal is not caused by the DEA to THF but rather to spurious water molecules: the energy dependence spectrum of the $\mathrm{O}^{-}$anion has basically the same shape as the ion yield of $\mathrm{O}^{-}$coming from DEA to water [44]. It is highly improbable that a signal with the same shape would originate from the electron collisions with THF, since the $11.5 \mathrm{eV}$ resonance is not visible in any other THF fragment. Water has several orders of magnitude larger DEA cross section [45], the present signal corresponds to approximately $0.5 \%$ impurity of water in our sample. The water impurity is also a reason for the $\mathrm{H}^{-}$signal visible in the spectrum of deuterated tetrahydrofuran in Fig. 1. Similarly as in the THF spectrum, this peak has a shoulder at short flight times caused by the heavy-ion settings of the ion lens.

\subsection{Total DEA cross section}

Fig. 3 shows the sum of our partial cross sections compared the total negative ion cross section of Aflatooni et al. [32]. Their data were obtained using a total ion collection tube, thus at electron energies larger than the ionization energy, a formation of positive ions sets in and interferes with the negative ion signal, causing the abrupt cutoff of the negative cross section. For the sake of the comparison, the spectrum of Aflatooni has been shifted by $360 \mathrm{meV}$ towards higher electron energy. Even though the inaccuracies of the energy scale calibration of the trochoidal monochromator on the order of tens of meV are expectable [46], the present discrepancy is relatively large and we do not know the reason for it. It should be noted, that our present energy position of the strongest mass 41 fragment agrees excellently both with Sulzer et al. and Ibanescu et al. Apart from the issue in the energy-position of the resonances, the agreement of the present total cross sections with 


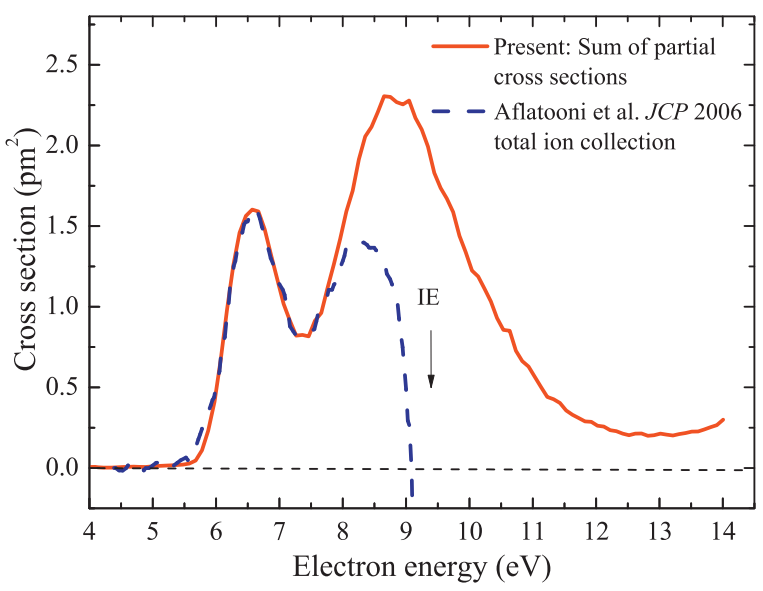

Fig. 3. Comparison of the sum of present partial cross sections to total cross section from Aflatooni et al. [32]. The vertical arrow shows the ionization potential of THF [43].

the total cross section of Aflatooni et al. is excellent up to the electron energy of approx. $8.5 \mathrm{eV}$. It should be noted that such high level of agreement is up to some level fortuitous in the view of the error bars of the both experiments ( $\pm 25 \%$ of the present values). The total ion collection data are lower than the present values above $8.5 \mathrm{eV}$. The ionization potential of THF is $9.42 \mathrm{eV}$ [43]. We can only speculate, what causes the decrease of the negative ion signal below this value. The cross sections for positive ionization are usually orders or magnitude larger than present DEA cross sections. The positive signal could be caused, for example, by even a very weak shoulder of high-energy electrons in the electron-energy distribution. Another option would be presence of an impurity with lower ionization energy in the sample - due to large ionization cross sections already a trace amount of such impurity would decrease the total negative signal considerably.

\subsection{Assignment of DEA bands}

Spectra of all the anionic fragments in Fig. 2 show three main bands centered around the electron energies of $6.6 \mathrm{eV}, 7.7 \mathrm{eV}$ and $8.7 \mathrm{eV}$. The dominant fragment originating from the first and the third band is $\mathrm{H}^{-}$, the dominant fragment appearing at the position of the second band is $\mathrm{C}_{3} \mathrm{H}_{5}{ }^{-}$. In the energy range of interest (above $6 \mathrm{eV}$ ) two classes of resonances have been suggested to occur in THF: one-particle shape resonances or core-excited (Feshbach) resonances.

The shape resonances in this energy range were identified theoretically by Trevisan et al. [23] at $8.6 \mathrm{eV}$ and by Winstead and McKoy [22] at 8.3 and $13 \mathrm{eV}$. Also, experiments on vibrational excitation [14] showed shape resonances at 6.2 and $10.8 \mathrm{eV}$. However, these resonances are very broad (few $\mathrm{eV}$ ), which is a common feature for shape resonances in organic molecules in this energy range. They usually arise from the electron occupation of the $\sigma^{*}$ orbitals associated with $\mathrm{C}-\mathrm{H}$ and $\mathrm{C}-\mathrm{C}$ bonds and due to their large energy width they are not expected to lead to detectable DEA signal. Their short autodetachment lifetime usually causes that the electron detaches before atomic nuclei move to such configuration in which the electronic state becomes a bound state, i.e., where one of the fragments becomes a stable anion.

The more suitable candidates for precursor resonant states in this energy range are core-excited Feshbach resonances. A Feshbach resonance has a cationic core and a double occupation of Rydberg-like orbitals. They are thus closely related to Rydberg and cationic states, this relation is demonstrated in the top panel of Fig. 4. The Rydberg (parent) states which are visible in VUV and
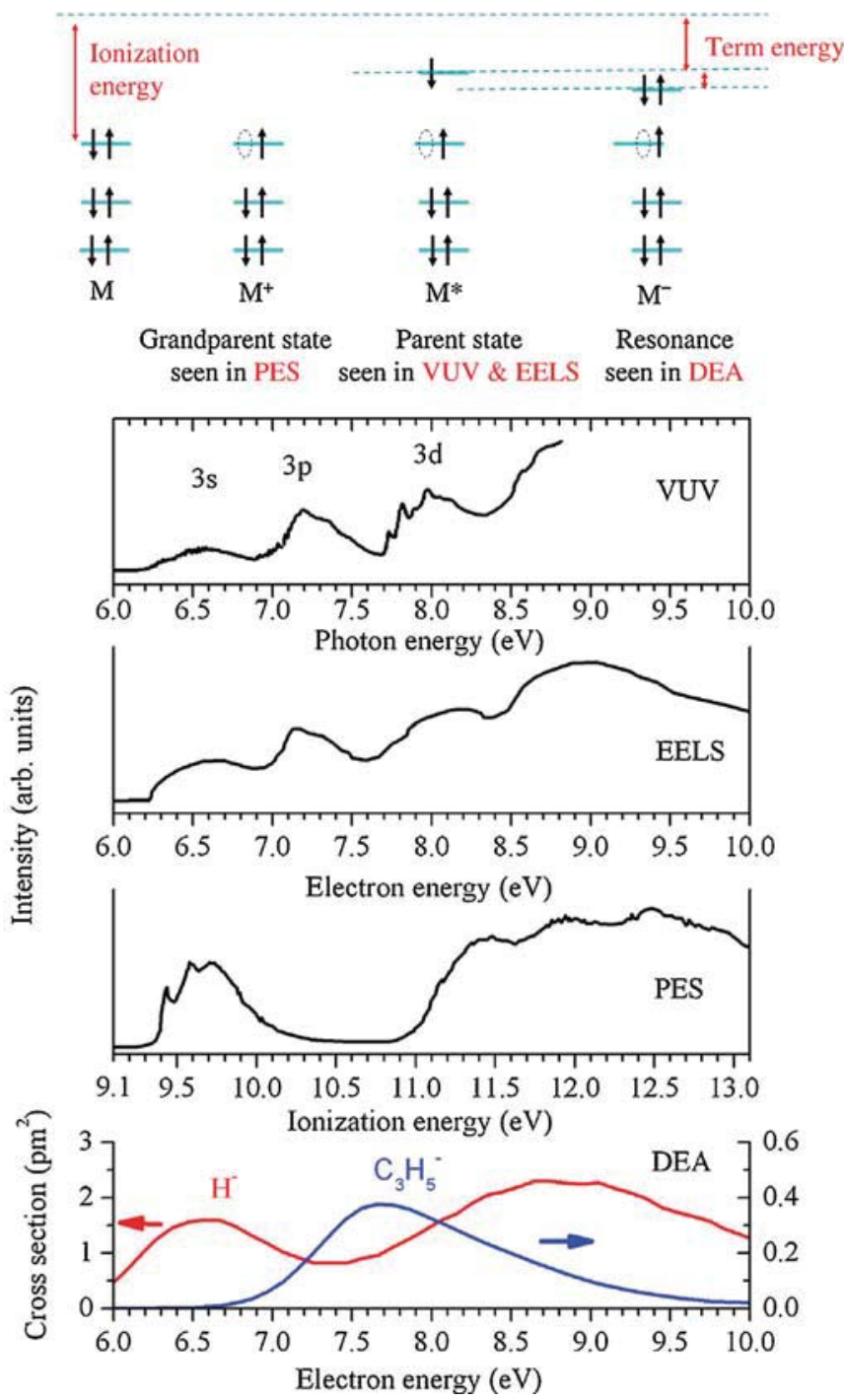

Fig. 4. Upper panel: schematic representation of the relation between VUV, EELS, PES and DEA spectra (see text for details). Graphs: (a) VUV spectrum of THF taken from Giuliani et al. [21], (b) EELS spectrum taken from Do et al. [20], (c) PES spectrum from Giuliani et al. [21], and (d) present DEA cross sections for $\mathrm{H}^{-}$and $\mathrm{C}_{3} \mathrm{H}_{5}$ fragments. The scale of the PES spectrum (c) is shifted by $3.1 \mathrm{eV}$ as explained in the text.

EELS (electron-energy loss spectroscopy) spectra are shifted with respect to the cation (grandparent) states, visible in the photoelectron spectra (PES), by a term energy. The resonance is further stabilized by the electron affinity of the parent Rydberg state which is typically $0.3-0.5 \mathrm{eV}$ for the $s^{2}$ configuration [47]. Recent work on excited electronic states of neutral and ionic states of THF by Guiliani et al. [21] greatly facilitates the assignment of resonances. Fig. 4 shows the VUV, EELS and PES spectra together with present cross sections for the two strongest DEA fragments. The high-level $a b$ initio calculations from Ref. [21] showed that all the electronically excited states of THF have Rydberg character. The first VUV and EELS band between 6 and $6.9 \mathrm{eV}$ is due to transition to 3s Rydberg state, the band is broadened by rich vibrational progressions and by the fact that the two THF conformers (with $C_{2}$ and $C_{s}$ geometries) have vertical excitation energies separated by $0.25 \mathrm{eV}$. The second band contains a mixture of $3 p$ and $3 d$ states, the third band consists of progression of $3 d$ states. All three bands are visible also in EELS spectra. In the PES spectrum the first band corresponds to the hole in the $9 b$ molecular orbital, comparison with the VUV spectrum shows that the term energy of the corresponding Rydberg state 
$(9 b)^{-1} 3 s$ is $3.1 \mathrm{eV}$. The scale of the PES spectrum in Fig. 4 starts at $9.1 \mathrm{eV}$, by $3.1 \mathrm{eV}$ higher than the scale of VUV and EELS spectra. If one then assumes $0.2-0.3 \mathrm{eV}$ as stabilization energy of the Feshbach electron pair, then the first band of the PES spectrum overlaps nicely with the first $\mathrm{H}^{-}$DEA band, and the electronic configuration of the resonance would be $(9 b)^{-1} 3 s^{2}$. The $8.7 \mathrm{eV} \mathrm{H}^{-}$DEA peak overlaps with the second broad band in the PES spectrum. This band corresponds to holes in $11 a, 10 a, 8 b$, and $9 a$ orbitals. The Feshbach resonances responsible for the DEA $\mathrm{H}^{-}$thus correspond to a $3 s^{2}$ electron pair around ionic core with the hole in one or several of these orbitals.

It is worth noticing that the PES spectrum has to be shifted only by approx. $3.3 \mathrm{eV}$ in order to overlap with the DEA bands. Often, this difference is assumed to be $4.5 \mathrm{eV}$ [40]. This assumption is based on an usual term energy of $4 \mathrm{eV}$ and stabilization energy of the $s^{2}$ pair of $0.5 \mathrm{eV}$ [47]. These values which are often considered to be the same for all molecules because of the weak interaction of the diffuse Rydberg orbital with the cationic core. However, for molecules with larger alkyl chains, the term energy is decreased due to the hindered approach of the Rydberg electron to the cationic core, thus decreasing the attraction energy and decreasing the term energy $[48,49]$. An extreme example was shown in the recent study of the TEMPO (2,2,6,6-Tetramethylpiperidine-N-oxyl) [50] where the shift between DEA and PES bands was only $2.1 \mathrm{eV}$. In the present case we have the advantage of knowing the shift between PES and VUV spectra to be $3 \mathrm{eV}$ due to the high-resolution data of Giuliani et al. [21].

The assignment of the $7.6 \mathrm{eV}$ band leading to the production of the $\mathrm{C}_{3} \mathrm{H}_{5}{ }^{-}$fragment is a puzzle. It does not overlap with any band in the shifted PES spectrum, so it is not possible to do an assignment as a Feshbach resonance with an electron pair in a $3 s$ orbital. In principle, one could speculate that it could be a Feshbach resonance with an electron pair in a $3 p$ orbital, or with a $3 s 3 p$ pair. Such resonances are seen in the transmission spectra, however, the stabilization energy of such electron pair is much lower, in the order of $0.01-0.1 \mathrm{eV}$ [47]. Thus their lifetime is expected to be too short to lead to observable DEA signals. This is a similar situation, as with the high-energy shape resonances mentioned above: their energies overlap with the DEA bands, however, such assignment is improbable due to a short lifetime towards autodetachment. For completeness, it should be mentioned that in this energy range DEA bands have also been found which originate from resonances where an $s$-like electron is bound to the singlet valence excited state. Some examples are norbornadiene, cyclopentadiene or $\mathrm{SO}_{2}$ [51]. However, no viable precursor excited states have been identified in THF [21].

\subsection{Note on the metastability}

Ibanescu et al. [31] studied DEA bond-breaking in a range of linear ethers and found that Feshbach resonances lead to very efficient cleavage of the $\mathrm{C}-\mathrm{O}$ bond. Since THF is a cyclic ether, they suggested that in the first step the $\mathrm{C}-\mathrm{O}$ bond is breaking as well, leading to an open-chain $\mathrm{THF}^{-}$parent anion. Since the energy released upon the ring-opening is stored in the internal degrees of freedom, this anion subsequently undergoes delayed dissociation.

In a time-of-flight setup, if such a delayed dissociation happens during the acceleration stage, before $\mathrm{THF}^{-}$reaches the drift region, the anions are detected between the flight-time of the parent anion and the anion into which it fragments. This effect has been used to estimate the lifetime of vibrationally hot $\mathrm{SF}_{6}{ }^{-}$[33] or dinitrobenzene anions [34]. In our setup the corresponding metastability time window is $0.2 \mu \mathrm{s}<t<1 \mu \mathrm{s}$. The lowest value corresponds to the delay between the electron pulse and the ion-extraction pulse, the largest delay to the time point when ions enter the drift tube. We have detected neither any remains of the $\mathrm{THF}^{-}$signal nor the signal corresponding to a metastable decay. This does not mean that the process suggested by Ibanescu et al. is not correct: due to high excess energy it probably happens on a faster time-scale. The only peak with slightly non-standard shape is that of $\mathrm{CH}_{2}{ }^{-}$which has a tail towards longer flight times. This indicates that it is in fact partly formed during the early stages of the acceleration.

Sulzer et al. [8] report occurrence of $\mathrm{THF}^{-}$anion, however, at lower electron energies, around $2 \mathrm{eV}$. It has been pointed out by Neumark and co-workers [52] that the $\mathrm{THF}^{-}$signal might have been caused by a small presence of clusters in the sample: the parent ion is stabilized in the DEA to a cluster, since the neighboring molecules act as a heat bath. The clustering might have been enhanced in the experiments of Sulzer et al. since they have used a molecular beam target, while in other studies, including the present one, stagnant gas target was used.

\section{Conclusions}

In conclusion, we have investigated the dissociative electron attachment in tetrahydrofuran. The present experiments using a time-of-flight setup revealed much richer fragmentation patterns than previously reported. The partial absolute DEA cross sections are very low, the dominant fragment $\mathrm{H}^{-}$has a peak cross section of $2.3 \mathrm{pm}^{2}$, the weakest detected fragment $\mathrm{C}_{3} \mathrm{H}_{3}{ }^{-}$has the peak cross section $0.002 \mathrm{pm}^{2}$. The fragmentation towards the most abundant heavy fragment is not driven by asymptotic energetics but rather by fast dissociative dynamics of the resonant anion. The spectra reveal three main DEA bands centered around $6.6 \mathrm{eV}, 7.7 \mathrm{eV}$ and $8.7 \mathrm{eV}$. Based on the comparison with the excited states of the neutral and ionic THF, the first and the last DEA band can be assigned to the Feshbach resonances corresponding to the holes in specific molecular orbitals and a $(3 s)^{2}$ electron pair. The sum of present partial cross section agrees with previous data of the total DEA cross sections [32] very well in the energy range up to $8.5 \mathrm{eV}$. Additionally, we have ruled out metastable decay processes happening in the $0.2 \mu \mathrm{s}<t<1 \mu \mathrm{s}$ time window.

\section{Acknowledgements}

This work is part of the project No. PZ00P2_132357 of the Swiss National Science Foundation. A.R. M. acknowledges support from the SNSF short international visit grant No. IZKOZ2_143984 and by the Ministry of Education, Science and Technological Development of Republic of Serbia under project No. 17020.

\section{References}

[1] B. Boudaiffa, P. Cloutier, D.J. Hunting, M.A. Huels, L. Sanche, Resonant formation of DNA strand breaks by low-energy (3 to $20 \mathrm{eV}$ ) electrons, Science 287 (2000) 1658.

[2] G. Hanel, B. Gstir, S. Denifl, P. Scheier, M. Probst, B. Farizon, M. Farizon, E. Illenberger, T.D. Märk, Electron attachment to uracil: effective destruction at subexcitation energies, Phys. Rev. Lett. 90 (2003) 188104.

[3] S. Denifl, S. Ptasinska, G. Hanel, B. Gstir, P. Scheier, M. Probst, B. Farizon, M. Farizon, S. Matejcik, E. Illenberger, T.D. Märk, Electron attachment to uracil, thymine and cytosine, Phys. Scr. T110 (2004) 252.

[4] S. Ptasinska, S. Denifl, P. Scheier, T.D. Märk, Inelastic electron interaction (attachment/ionization) with deoxyribose, J. Chem. Phys. 120 (2004) 8505.

[5] S. Ptasinska, S. Denifl, P. Candori, S. Matejcik, P. Scheier, T.D. Märk, Dissociative electron attachment to gas phase alanine, Chem. Phys. Lett. 403 (2005) 107.

[6] S. Ptasinska, S. Denifl, P. Scheier, E. Illenberger, T.D. Märk, Bond- and siteselective loss of $\mathrm{H}$ atoms from nucleobases by very-low-energy electrons $(<3 \mathrm{eV})$, Angew. Chem. Int. Ed. 44 (2005) 6941.

[7] P.D. Burrow, G.A. Gallup, A.M. Scheer, S. Denifl, S. Ptasinska, T.D. Märk, P. Scheier, Vibrational Feshbach resonances in uracil and thymine, J. Chem. Phys. 124 (2006) 124310 .

[8] P. Sulzer, S. Ptasinska, F. Zappa, B. Mielewska, A. Milosavljević, P. Scheier, T.D. Märk, I. Bald, S. Gohlke, M.A. Huels, E. Illenberger, Dissociative electron attachment to furan, tetrahydrofuran, and fructose, J. Chem. Phys. 125 (2006) 044304. 
[9] A. Zecca, C. Perazolli, M. Brunger, Positron and electron scattering from tetrahydrofuran, J. Phys. B: Atom. Mol. Phys. 38 (2005) 2079.

[10] P. Mozejko, E. Ptasinska-Denga, A. Domaracka, C. Szmytkowski, Absolute total cross-section measurements for electron collisions with tetrahydrofuran, Phys. Rev. A 74 (2006) 012708.

[11] M. Fuss, A. Munoz, J.C. Oller, F. Blanco, D. Almeida, P. Limao-Vieira, T.P.D. Do, M.J. Brunger, G. Garcia, Electron-scattering cross sections for collisions with tetrahydrofuran from 50 to 5000 eV, Phys. Rev. A 80 (2009) 052709

[12] A.R. Milosavljević, A. Giuliani, D. Šević, M. Hubin-Franskin, B.P. Marinković, Elastic scattering of electrons from tetrahydrofuran molecule, Eur. Phys. J. D35 (2005) 411.

[13] M. Dampc, A.R. Milosavljević, I. Linert, B.P. Marinković, M. Zubek, Differential cross sections for low-energy elastic electron scattering from tetrahydrofuran in the angular range $20^{\circ}-180^{\circ}$, Phys. Rev. A 75 (2007) 042710.

[14] M. Allan, Absolute angle-differential elastic and vibrational excitation cross sections for electron collisions with tetrahydrofuran, J. Phys. B: Atom. Mol. Phys. 40 (2007) 3531.

[15] C.J. Colyer, V. Vizcaino, J.P. Sullivan, M.J. Brunger, S.J. Buckman, Absolute elastic cross-sections for low-energy electron scattering from tetrahydrofuran, New J. Phys. 9 (2007) 41

[16] W.Y. Baek, M. Bug, H. Rabus, E. Gargioni, B. Grosswendt, Differential elastic and total electron scattering cross sections of tetrahydrofuran, Phys. Rev. A 86 (2012) 032702.

[17] L. Chiari, L. Anderson, W. Tattersall, J.R. Machacek, P. Palihawadana, C. Makochekanwa, J.P. Sullivan, G. Garcia, F. Blanco, R.P. McEachran, M.J. Brunger, S.J. Buckman, Total, elastic, and inelastic cross sections for positron and electron collisions with tetrahydrofuran, J. Chem. Phys. 138 (2013) 074301

[18] M.G.P. Homem, R.T. Sugohara, I.P. Sanches, M.T. Lee, I. Iga, Cross sections for elastic electron collisions with tetrahydrofuran, Phys. Rev. A 80 (2009) 032705.

[19] M. Dampc, I. Linert, A.R. Milosavljević, M. Zubek, Vibrational excitation of tetrahydrofuran by electron impact in the low energy range, Chem. Phys. Lett. 443 (2007) 17

[20] T.P.D. Do, M. Leung, M. Fuss, G. Garcia, F. Blanco, K. Ratnavelu, M.J. Brunger, Excitation of electronic states in tetrahydrofuran by electron impact, J. Chem. Phys. 134 (2011) 144302

[21] A. Giuliani, P. Limao-Vieira, D. Duflot, A. Milosavljevic, B.P. Marinkovic, S.V.N. Hoffmann, J. Delwiche, M.-J. Hubin-Franskin, Electronic states of neutral and ionized tetrahydrofuran studied by VUV spectroscopy and ab initio calculations, Eur. Phys. J. D51 (2009) 97.

[22] C. Winstead, V. McKoy, Low-energy electron scattering by deoxyribose and related molecules, J. Chem. Phys. 125 (2006) 074302.

[23] C.S. Trevisan, A.E. Orel, T.N. Rescigno, Elastic scattering of low-energy electrons by tetrahydrofuran, J. Phys. B: Atom. Mol. Phys. 39 (2006) L255.

[24] D. Bouchiha, J.D. Gorfinkiel, L.G. Caroh, L. Sanche, Low-energy electron collisions with tetrahydrofuran, J. Phys. B: Atom. Mol. Phys. 39 (2006) 975.

[25] M. Lepage, S. Letarte, M. Michaud, F. Motte-Tollet, M.-J. Hubin-Franskin, D. Roy, L. Sanche, Electron spectroscopy of resonance-enhanced vibrational excitations of gaseous and solid tetrahydrofuran, J. Chem. Phys. 108 (1998) 5980.

[26] D. Antic, L. Parenteau, M. Lepage, L. Sanche, Low-energy electron damage to condensed-phase deoxyribose analogues investigated by electron stimulated desorption of $\mathrm{H}^{-}$and electron energy loss spectroscopy, J. Phys. Chem. B 103 (1999) 6611.

[27] D. Antic, L. Parenteau, L. Sanche, Electron-stimulated desorption of $\mathrm{H}^{-}$from condensed-phase deoxyribose analogues: dissociative electron attachment versus resonance decay into dipolar dissociation, J. Phys. Chem. B 104 (2000) 4711.

[28] S.P. Breton, M. Michaud, C. Jäggle, P. Swiderek, L. Sanche, Damage induced by low-energy electrons in solid films of tetrahydrofuran, J. Chem. Phys. 121 (2004) 11240.

[29] C. Jäggle, P. Swiderek, S.P. Breton, L. Sanche, Products and reaction sequences in tetrahydrofuran exposed to low-energy electrons, J. Phys. Chem. B 110 (2006) 12512.
[30] Y. Park, H. Cho, L. Parenteau, A.D. Bass, L. Sanche, Cross sections for electron trapping by DNA and its component subunits I: condensed tetrahydrofuran deposited on Kr, J. Chem. Phys. 125 (2006) 074714.

[31] B.C. Ibanescu, O. May, M. Allan, Cleavage of the ether bond by electron impact: differences between linear ethers and tetrahydrofuran, Phys. Chem. Chem. Phys. 10 (2008) 1507.

[32] A. Aflatooni, A.M. Scheer, P.D. Burrow, Total dissociative electron attachment cross sections for molecular constituents of DNA, J. Chem. Phys. 125 (2006) 054301.

[33] K. Graupner, T. Field, A. Mauracher, P. Scheier, A. Bacher, S. Denifl, F. Zappa, T.D. Märk, Fragmentation of metastable SF_ ${ }^{6}$ ions with microsecond lifetimes in competition with autodetachment, J. Chem. Phys. 128 (2008) 104304.

[34] A. Mauracher, S. Denifl, A. Edtbauer, M. Hager, M. Probst, O. Echt, T.D. Märk.

[35] J. Fedor, O. May, M. Allan, Absolute cross sections for dissociative electron attachment to $\mathrm{HCl}, \mathrm{HBr}$, and their deuterated analogs, Phys. Rev. A 78 (2008) 032701.

[36] O. May, J. Fedor, M. Allan, Absolute cross sections for dissociative electron attachment to acetylene and diacetylene, Phys. Rev. A 77 (2008) 040701(R).

[37] O. May, J. Fedor, M. Allan, Isotope effect in dissociative electron attachment to acetylene, Phys. Rev. A 80 (2009) 012706.

[38] R. Dressler, M. Allan, Energy partitioning in the $\mathrm{O}^{-} / \mathrm{CO}_{2}$ dissociative attachment, Chem. Phys. 92 (1985) 449.

[39] R. Janečková, D. Kubala, O. Ma, J. Fedor, M. Allan, Experimental evidence on the mechanism of dissociative electron attachment in formic acid, Phys. Rev. Lett. 111 (2013) 213201, in press.

[40] B.C. Ibanescu, O. May, A. Monney, M. Allan, Electron-induced chemistry of alcohols, Phys. Chem. Chem. Phys. 9 (2007) 3163.

[41] R. Janečková, O. May, J. Fedor, Dissociative electron attachment to methylacetylene and dimethylacetylene: symmetry vs. proximity, Phys. Rev. A 86 (2012) 052702.

[42] V.M. Rayon, V.A. Sordo, Pseudorotation motion in tetrahydrofuran: an ab initio study, J. Chem. Phys. 122 (2005) 204303.

[43] J.L. Holmes, F.P. Lossing, Ionization energies of homologous organic compounds and correlation with molecular size, Org. Mass. Spectrom. 26 (1991) 537.

[44] J. Fedor, P. Cicman, B. Coupier, S. Feil, M. Winkler, K. Gluch, J. Husarik, D. Jaksch, B. Farizon, N.J. Mason, P. Scheier, T.D. Märk, Fragmentation of transient water anions following low-energy electron capture by $\mathrm{H}_{2} \mathrm{O} / \mathrm{D}_{2} \mathrm{O}$, J. Phys. B: Atom. Mol. Phys. 39 (2006) 3935.

[45] P. Rawat, V.S. Prabhudesai, G. Aravind, M.A. Rahman, E. Krishnakumar, Absolute cross sections for dissociative electron attachment to $\mathrm{H}_{2} \mathrm{O}$ and $\mathrm{D}_{2} \mathrm{O}$, J. Phys. B: Atom. Mol. Phys. 40 (2007) 4625.

[46] J. Fedor, M. Cingel, J.D. Skalný, P. Scheier, T.D. Märk, M. Čížek, J. Kolorenč, J. Horáček, Dissociative electron attachment to $\mathrm{HBr}$ : a temperature effect, Phys. Rev. A 75 (2007) 022703.

[47] D. Spence, Prediction of low energy molecular Rydberg states from Feshbach resonance spectra, J. Chem. Phys. 66 (1977) 669.

[48] M.B. Robin, Higher Excited States of Polyatomic Molecules, Academic Press, New York, 1974

[49] B.C. Ibanescu, M. Allan, Selective cleavage of the $\mathrm{C}-\mathrm{O}$ bonds in alcohols and symmetric ethers by dissociative electron attachment, Phys. Chem. Chem. Phys. 11 (2009) 7640.

[50] D. Kubala, K. Regeta, R. Janečková, J. Fedor, S. Grimme, A. Hansen, P. Nesvadba, M. Allan, The electronic structure of TEMPO, its cation and anion, Mol. Phys. 111 (2013) 2033.

[51] V.I. Khvostenko, A.S. Vorobyov, O.G. Khvostenko, Inter-shell resonances in the interactions of electrons and polyatomic molecules, J. Phys. B: Atom. Mol. Phys. 23 (1990) 1975.

[52] R.M. Young, M.A. Yandell, M. Niemeyer, D.M. Neumark, Photoelectron imaging of tetrahydrofuran cluster anions (THF) $n^{-}(1<n<100)$, J. Chem. Phys. 133 (2010) 154312. 